\title{
HEGEL Y LA HISTORIOGRAFÍA FILOSÓFICA
}

\author{
Andrés MARTÍNEZ LORCA \\ Departamento de Filosofia. UNED
}

Resumen: La historiografia filosófica de Hegel se sustenta sobre una sugestiva filosofia de la historia que puede caracterizarse como racionalista (la razón dirige la historia), providencialista (su providencialismo "al por mayor" concluye en una Teodicea de inspiración cristiana) y progresista (el progreso como motor de la historia).

En este estudio se presta especial atención a la concepción hegeliana de la historia de la filosofia, que gira en torno a los conceptos de desarrollo, Entwicklung, y de concreto, das Konkrete. La filosofia es asi sistema en desarrollo, y lo es también la historia de la filosofia: coinciden, por tanto, el desarrollo histórico y el desarrollo lógico de la filosofia. Entre las tesis que Hegel deduce de tal fundamento, debemos destacar ésta: toda filosofia es la filosofia de su tiempo, un eslabón en la gran cadena del desarrollo espiritual.

Finalmente, se sintetizan las criticas de Marx, Dilthey y Croce a la concepción hegeliana de la filosofia y de su historia.

Abstract: Hegel's philosophical historiography is based on his interesting philosophy of history that can be described as rationalist (reason directs history), providencialist (his «wholesale» providencialism concludes in a Theodicy of christian inspiration) and progressive (progress as a motor of history).

In this study special attention is paid to the hegelian conception of the history of philosophy, that is concentrated on the concepts of development, Entwicklung, and of concret, das Konkrete. Philosophy is so a system in development, and the same can be said of the history of philosophy: therefore the historical and the logical development of philosophy agree between them. Among the theses that Hegel deduces from such premises, this must be pointed up: every philosophy is the philosophy of its time, a link in the great chain of the intellectual development. 
Finally are summarized the criticisms from Marx, Dilthey and Croce against the hegelian conception of philosophy and of its history.

"Jede Philosophie ist Philosophie ihrer Zeit, sie ist Glied in der ganzen Kette der geistigen Entwicklung".

G.W.F. Hegel, Vorlesungen über die Geschichte der Philosophie, Einleitung.

\section{La Filosofia de la historia de Hegel}

Con Hegel la historiografía filosófica alcanza su mayoría de edad. Tras los islotes de Aristóteles y Averroes, tocamos, por fin, tierra firme. La contribución del pensador alemán es doble: como filósofo de la historia y como historiador de la filosofia. Analizaremos a partir de ahora una y otra.

Hegel dedicó a la reflexión sobre la historia una atención preferente, de la que dieron testimonio sus asiduos cursos sobre la materia en la Universidad. Fruto de esas clases fue la recopilación que llevó a cabo Eduard Gans, primer editor (en 1837) de las Vorlesungen iiber die Philosophie der Geschichte. La segunda edición (1840) y la tercera (1843) incluyeron diversos manuscritos del propio Hegel en forma de notas: ambas ediciones estuvieron al cuidado de Karl Hegel, hijo del gran maestro alemán. Una ampliación considerable representó la cuarta edición (Leipzig, 1917-1920), preparada por Georg Lasson, en la que se integraron un nuevo manuscrito de Hegel y varios cuadernos de apuntes tomados por alumnos de diferentes cursos académicos. La quinta y última edición alemana pretende dar una mayor unidad al texto anterior $\mathrm{e}$ incorpora también algunas adiciones; cuidada por Johannes Hoffmeister, vió la luz en 1955 por la misma editorial pero en nueva sede (Felix Meiner, Hamburgo). Afortunadamente, tenemos una excelente versión íntegra de la edición de Lasson, obra de José Gaos. Posteriormente, ha sido traducida también una parte de la edición de Hoffmeister ${ }^{1}$. El núcleo de la filosofía de la

1 G.W.F. Hegel, Lecciones sobre la filosofla de la historia universal traducción de José Gaos, Madrid, 1928; cito por la 4. ${ }^{2}$ edición, Madrid, 1974, que incluye un valiosísimo Prólogo de José Ortega y Gasset. Por otra parte, G.W.F. HEGEL, La Razón en la Historia, versión castellana de César Armando Gómez y nota introductoria de Antonio Truyol, Madrid, 1972, contiene solamente la "Introducción" a las Lecciones según el texto de Hoffmeister. 
historia hegeliana está contenido en la "Introducción» a las Vorlesungen, titulada muy expresivamente por los editores alemanes Die Vernunft in der Geschichte, y en ella nos concentraremos.

Es evidente que en Hegel confluyen muy distintas influencias que él se esfuerza en reelaborar. De un lado, el racionalismo filosófico con el que sintoniza a fondo. De otro, la Ilustración con cuyos ideales emancipatorios simpatizará desde su juventud. Asume también reflexivamente la tradición especulativa alemana que en los tiempos modernos arranca de Leibniz y se consolida en Kant, hasta llegar a representar él mismo la culminación del llamado «idealismo alemán". Y en el campo que más nos afecta ahora, se interesa vivamente por el historicismo cuyas ideas van germinando en la cultura ilustrada europea, primero silenciosamente con el italiano Vico y más tarde, de modo llamativo, con los franceses Voltaire y Montesquieu, y que ya contaba con dignos continuadores en suelo alemán como Herder y Wilhelm von Humboldt.

El punto de partida es, evidentemente, el mundo histórico. Pero desde el primer momento, Hegel intenta elevarse sobre los historiadores de oficio para hacer algo distinto, lo que él llama "historia filosófica", es decir, una filosofía de la historia. Así aparece escrito en un primer esbozo de 1822 con el que acertadamente, abre esta "Introducción" el editor Hoffmeister. Se distinguen aquí ${ }^{2}$ tres tipos de historia:

1. Historia original (o inmediata en la versión de Gaos). «Se trata de historiadores que han descrito, sobre todo, las acciones, acontecimientos y situaciones por ellos vividos, que estuvieron personalmente atentos a su espíritu". Podríamos llamarlos cronistas. Entre el escritor y las acciones que relata se da una "unidad de espíritu», una "comunidad de cultura». Heródoto, Tucídides y Jenofonte pertenecerían a este tipo.

2. Historia reflexiva. "Esta forma trasciende la actualidad en la que el historiador vive para tratar el pasado más lejano como actual en espiritu. Lo que en ella cuenta es la elaboración de los materiales históricos, y el espíritu que preside este trabajo de elaboración difiere del espiritu del contenidon. Se englobarían en este tipo la generalidad de los que consideramos historiadores; un

2 La Razón en la Historia, cit., pp. 17-37. Una reelaboración de este primer esbozo aparece incluída en la edición de Lasson después del texto de la "Introducción general«: véase Lecciones sobre la filosofia de la historia universal, cit., pp. 153-160. 
buen modelo antiguo sería Tito Livio. La historia reflexiva puede subdividirse a su vez, según Hegel, en historia global (pretende una visión de conjunto de un pueblo y debe poseer cierta unidad); historia pragmática (nos presenta «un mundo reconstruido mediante la reflexión", fin común a toda historia); e historia crítica (en realidad, teoría de la historia o metahistoria, que incluye también una crítica de las fuentes).

3. Historia filosífica. Relacionada directamente con la historia crítica, «su punto de vista es igualmente general, pero no se pliega a una esfera particular ni se deja separar abstractamente de los demás puntos de vistam. En este primer esbozo de 1822 se considera a la historia filosófica como «el objeto de nuestro curson. (Pero en el segundo esbozo de 1830, madurado ya su pensamiento sobre el problema de la historia, Hegel introduce una terminología más precisa, a la que nos atendremos en adelante: «el objeto de estos cursos es la filosofia de la historia universabi). A la concepción hegeliana de la filosofia de la historia, tal como se expone en las páginas siguientes de la "Introducción", prestaremos atención a partir de ahora.

Hegel comienza con la afirmación de que «la razón rige al mundo y que, por tanto, también la historia universal ha transcurrido racionalmenten. Bajo esta perspectiva racionalista late la pretensión de ignorar la superficie fenoménica de la historia, donde reina el azar y la arbitrariedad. Ortega vio bien que la ciencia histórica debe determinar lo que hay de constante y lo que hay de azaroso, evitando así convertir lo histórico en el reino de la casualidad, porque, en caso contrario, corre el riesgo de liquidar su intento científico, convirtiendo su objeto en inefable. El filósofo alemán, por su parte, somete la categoría de variación al fin último en sí y por sí. El "cambio de los individuos, pueblos y Estados, que existen en un momento... y enseguida desaparecen", queda relegado a lo inesencial de la historia. Lo esencial es, por el contrario, dirigir la historia con la especulación filosófica "tratándola como un material, y no dejándola tal como es, sino disponiéndola con arreglo al pensamiento y construyendo a priori una historias. Por eso define su filosofia de la historia como "la consideración pensante de la historia». Hegel es rotundo en la determinación de este principio de la filosofía de la historia. «Es necesario llevar a la historia la fe y el pensamiento de que el mundo de la voluntad no está entregado al acaso. Damos por supuesto, como verdad, que en los acontecimientos de los pueblos domina un fin último, que en la historia universal hay una razón —no la razón de un sujeto particular, sino la razón divina y absoluta-. (...) Pero esta fe universal, la creencia de que la historia universal es un producto de la razón eter- 
na y de que la razón ha determinado las grandes revoluciones de la historia, es el punto de partida necesario de la filosofía en general y de la filosofía de la historia universal» ${ }^{3}$.

Otro rasgo definitorio de la filosofía hegeliana de la historia es el providencialismo. Hegel conecta con la esfera de la religión y desarrolla este punto partiendo de la creencia en una providencia universal. Se muestra insatisfecho tanto "con aquella fe que administra la Providencia al por menor" (es decir, la religiosidad popular en sus manifestaciones cotidianas), como "con la fe meramente abstracta e indeterminada que se satisface con la fórmula general de que hay una Providencia que rige el mundo, pero sin querer entrar en lo determinado y concreto" (o sea, la propia de una consciencia ilustrada de la época). En su propuesta alternativa de hacer patente en la historia una providencia al por mayor y de absorber, depurándola, a la religión ("la filosofia tiene que hacerse cargo del contenido de la religión, incluso contra algunas formas de teologían), se evidencia la matriz religiosa del idealismo alemán y esa tendencia a la desmesura tan característica del espíritu germánico. Este providencialismo hegeliano acaba lógicamente en una explícita Teodicea. "Nuestra consideración es, por tanto, una Teodicea, una justificación de Dios, como la que Leibniz intentó metafísicamente, a su modo, en categorías aún abstractas e indeterminadas». La deuda con los cristianos por haber recibido de ellos «la clave de la historia universal", es reconocida en estas páginas ${ }^{4}$.

Como antes lo azaroso, Hegel hace desaparecer ahora de la historia lo individual. Lo que importa es la totalidad encarnada en el «espíritu del pueblo» (Volksgeist): "en el elemento de la historia universal no tenemos que habérnoslas con el individuo particular, ni con la limitación y referencia a la individualidad particular. El espíritu, en la historia, es un individuo de naturaleza universal pero a la vez determinada, esto es: un pueblo en general. Y el espíritu de que hemos de ocuparnos es el espíritu del pueblo. (...) Las individualidades, por tanto, desaparecen para nosotros y son para nosotros las que vierten en la realidad lo que el espíritu del pueblo quiere» ${ }^{5}$. La forma de esta totalidad es orgánica. El espíritu del pueblo es concebido como "un individuo natural» que florece, madura y muere. Tal concepción organicista le permite a Hegel buscar

\footnotetext{
Lecciones sobre la filosafla de la historia universah pp. 44 y 55. Véanse también pp. 41-48.

Ibid., pp. 50-57.

Ibid. pp. 65-66.
} 
la reconciliación de las clases sociales en la totalidad del Volksgeist. Más aun, someter la moralidad del hombre concreto a las determinaciones de su clase social. "La moralidad del individuo consiste, además, en cumplir los deberes de su clase. Y esto es cosa fácil de saber; los deberes están determinados por la clase, ${ }^{6}$. Este aspecto, habitualmente poco comentado, del pensamiento hegeliano ofrece, sin duda, muchos atractivos a cualquier régimen político conservador. Y la historia reciente europea y española podría presentarnos tantos ejemplos prácticos como para cansarnos... Reaparece aquí claramente la concepción de la justicia expuesta en la República, que Platón cubre con un velo de pudor al explicar el origen de las clases sociales mediante un oscuro mito fenicio.

El espíritu del pueblo se realiza en el Estado (Staat), que se convierte así en el objeto de la historia. La voluntad objetiva y la subjetiva se identifican en el Estado, constituido en "orbe moral", en "la vida moral realizada". Él es también la manifestación de la universalidad tan buscada por Hegel. "Lo universal...debe ser una realidad y como tal existe en el Estado. Es lo que tiene validez" 7 . Con una brillante fórmula que nos remite al racionalismo del punto de partida, llega a escribir: “El Estado es la razón en la tierra» ${ }^{8}$.

En su consideración del desarrollo humano, Hegel descubre el progreso como motor de la historia. Este pensamiento ilustrado marca uno de los rasgos de su filosofía de la historia. Para él, la naturaleza es un círculo, una repetición de lo mismo. "En la naturaleza no sucede nada nuevo bajo el sol; por eso el espectáculo multiforme de sus transformaciones produce hastío" ${ }^{9}$. Por el contrario, el espíritu humano avanza en un movimiento infinito atravesando una serie de fases determinadas. "En la naturaleza, la especie no hace ningún progreso; en el espíritu empero, toda transformación es progreso. (...) La historia universal es el progreso en la conciencia de la libertad —un progreso que debemos conocer en su necesidad ${ }^{10}$. Esta concepción de la libertad como consciencia de la necesidad, de origen estoico y que recogería Spinoza en su Etica, se opone radicalmente a la teoría epicúrea de la libertad. Por eso Hegel

6 Ibid., p. 89, cursiva mía.

7 Ibid., p. 102. Véanse también sobre la naturaleza del Estado pp. 100-104.

$8 \quad$ Ibid., p. 123.

9 Ibid., p. 127. La ceguera de Hegel ante la evolución biológica es total.

$10 \quad$ Ibid., pp. 138 y 68 ; la cursiva es mía. 
no desaprovecha la ocasión de reprocharle a Epicuro que atribuya dem Zufall alle Ereignisse ("todos los sucesos al azar»), aunque se consuela al comprobar que tal teoría representa una excepción en la historia de la filosofía.

Un optimismo desbordante, una fe ciega en el progreso, impregnan esta filosofía de la historia. Los ideales de una burguesía triunfante que identificaba su propia emancipación política con la emancipación general de la humanidad, son también los ideales de Hegel. Pero a veces esa imparable marcha de la historia descubre su lado oscuro, las grietas provocadas por la miseria real. Los caminos de la libertad se han abierto paso entre el dolor de los hombres de carne y hueso. "Si consideramos este espectáculo de las pasiones y fijamos nuestros ojos en las consecuencias históricas de su violencia, de la irreflexión que acompaña, no sólo a ellas, sino también, y aun preferentemente, a los buenos propósitos y rectos fines; si consideramos el mal, la perversidad y la decadencia de los más florecientes imperios que el espíritu humano ha producido; si miramos a los individuos con la más honda piedad por su indecible miseria, hemos de acabar lamentando con dolor esta caducidad y - ya que esta decadencia no es sólo obra de la naturaleza, sino de la voluntad humana - con dolor también moral, con la indignación del buen espíritu, si tal existe en nosotros" ${ }^{11}$.

Pero este dolor es bien pasajero y no encierra, en verdad, más que un lamento retórico. Hegel se consuela pronto cuando reflexiona sobre el fin último al que sirve «este enorme sacrificio». Según su lapidaria frase, die Weltgeschichte ist das Weltgericht. Esta "consumación de la historia» es la que da sentido al proceso histórico. Por otra parte, para hacer progresar la marcha de la idea, "la razón hace que las pasiones obren por ella y que aquello mediante lo cual la razón llega a la existencia, se pierda y sufra daño» ${ }^{12}$. Es lo que llama Hegel con expresión feliz die List der Vernunft, "el ardid de la razón". No hay, por tanto, que detenerse a meditar sobre la miseria del hombre concreto, pues ello conduciría a "complacerse melancólicamente sobre las vanas e infecundas sublimidades de aquel resultado negativo». Tampoco cabe desconfiar del inexorable triunfo de la razón en la historia.

Encontramos también en las Lecciones sobre la filosofia de la historia otro género de reflexiones. En su implacable crítica al positivismo de los historia-

11 Ibid., pp. 79-80.

12 Ibid., p. 97. 
dores de oficio para quienes la ciencia histórica consistiría en el conocimiento de los datos, Hegel subraya que sin teoria no puede haber historia. Esta va más allá de un simple agregado de datos. Incluso la semántica del término denota la unión de un doble sentido. «La palabra historia reúne en nuestra lengua el sentido objetivo y el subjetivo: significa tanto historiam rerum gestarum como las res gestas mismas, tanto la narración histórica como los hechos y acontecimientos. Debemos considerar esta unión de ambas acepciones como algo más que una casualidad externa»" ${ }^{13}$.

Partiendo de la tesis metacientífica de Hegel, Ortega y Gasset llegó a desarrollar de modo autónomo este núcleo conceptual. Es lo que llamó historiologia, es decir, teoría de la historia. Ortega comienza reconociendo que la filosofía hegeliana de la historia en su uintento de construir el contenido de la historia mediante categorías sensu stricto filosóficas", se convirtió en una metafisica de la historia ${ }^{14}$. Y no le extraña, en consecuencia, el recelo de la "escuela histórica «ante ese «intolerable imperialismom. Tras este paso inicial, el horizonte intelectual que describe es desolador. Por un lado, «no ha habido una metahistoria que defina lo real histórico in genere, que lo analice en sus categorías primarias». Y por otro, la pobreza teórica de los estudios históricos resulta alarmante: «Es inaceptable en la historiografía y filología actuales el desnivel existente entre la precisión, usada al obtener o manejar los datos, y la imprecisión, más aún, la miseria intelectual en el uso de las ideas constructivas" ${ }^{15}$. Distanciada, pues, tanto del idealismo hegeliano como del positivismo histórico, la historiología no intenta ser historiografía, o sea, reflexión metodológica sobre la historia rerum gestarum, sino análisis de la propia realidad histórica, de las res gestae. La propuesta orteguiana, a pesar de algunas insuficiencias en su planteamiento general, y a pesar también de no haber superado la fase embrionaria de este Prólogo, encierra sin duda una rica perspectiva del mundo histórico que no debería desaprovecharse en el futuro. "No es posible, pues, reducir la historia al ingrediente inferior de los constitutivos de toda ciencia empirica. A las técnicas inferiores con que rebusca los datos es preciso aniadir y anteponer otra técnica de rango incomparablemente más elevado: la ontología de la realidad histórica, el estudio a priori de su estructura esencial. Sólo esto puede transformar a la historia en ciencia, es decir, en reconstrucción de lo real

\footnotetext{
$13 \quad$ Ibid, p. 137.

14 Ibid, p. 29. El texto íntegro del Prólogo de Ortega ocupa las pp. 15-32.

15 Ibid., pp. 28-22.
} 
mediante una construcción a priori de lo que en esa realidad —en este caso la vida histórica - haya de invariante» ${ }^{16}$.

\section{Hegel, historiador de la filosofía}

Hegel investigó a fondo la historia de la filosofía. De hecho, sus cursos sobre esta materia fueron los que más veces ofreció, reelaborándolos, en diversas universidades alemanas. Poco después de su muerte, se reconstruyeron tomando como base los apuntes de algunos alumnos y varios manuscritos del propio Hegel. La publicación de estas lecciones, las Vorlesungen über die Geschichte der Philosophie, hizo época. No podemos entrar ahora en el examen de la historiografía hegeliana en general. Lo que nos importa es analizar su concepto de historia de la filosofía, que por primera vez es abordado de modo explícito en estas páginas y más en concreto en la «Introducción» a las lecciones.

Antes de ello, hagamos una breve historia del texto, ya que, como he señalado antes, el grueso del mismo no procede de Hegel sino de los apuntes tomados en clase. La primera edición fue preparada por Karl Ludwig Michelet y se editó en $1833^{17}$. En el texto se integraban fragmentos ya elaborados y borradores del mismo Hegel, junto a diversos apuntes que representaban un desarrollo vivo de los esquemas previos. El esqueleto lo formaba el cuaderno de Jena, redactado por el maestro para el primero de los cursos sobre historia de la filosofía (1805-1806). Michelet publicó una segunda edición entre los años 1840-1844, en la que introdujo algunas modificaciones y supresiones. Hermann Glockner preparó ya en nuestro siglo una tercera edición dentro de la Jubiläumausgabe, basándose en el texto de Michelet. Frente al objetivo de sistematicidad integral que intentaba disimular la operación de ensamblaje de textos (línea de trabajo seguida por Michelet), Johannes Hoffmeister procuró reflejar la heterogeneidad de los elementos mezclados, y las alteraciones producidas en el desarrollo del pensamiento de su autor, añadiendo también nuevos manuscritos y apuntes. Esta nueva edición está considerada por todos los crí-

16 Ibid., p. 28, cursiva mía.

17 Vorlesungen über die Geschichte der Philosophie, editor K. L. Michelet, Berlín, 1833, 3 vols. Disponemos de una buena versión castellana de la obra completa: Lecciones sobre la historia de la filosofia, traducción de Wenceslao Roces, México D.F., 1955, 3 vols. 
ticos como la mejor disponible ${ }^{18}$. El único inconveniente que tiene es que es incompleta, pues se reduce a la Einleitung o "Introducción" a las lecciones (limitación que no nos afecta ahora, dado el carácter conceptual y no historiográfico de nuestro análisis).

Comencemos, como hace Hoffmeister, con el texto más antiguo de la Einleitung, el manuscrito de Heidelberg fechado en 1816, con el que Hegel presentó sus lecciones de historia de la filosofia en aquella universidad, y cuyo carácter embrionario y casi programático podemos comprobar si lo comparamos con desarrollos posteriores, y en particular con el famoso discurso de Berlín de 1818. El racionalismo que inspira la concepción hegeliana y que representa una ruptura con la epistemología de Kant, aparece ya en las líneas iniciales donde señala la fe en la razón como condición primera de la filosofia: «Der Mut der Wahrheit, der Glaube an die Macht des Geistes ist die erste Bedingung der Philosophien ${ }^{19}$.

A continuación, Hegel introduce la cuestión metodológica de la importancia del concepto de filosofía para la propia historia de la filosofía. Esta conexión entre concepto e historia muestra su máxima utilidad precisamente en el caso de la filosofía. De ahí su crítica a la historiografía filosófica anterior a la que reprocha en un conocido símil su incapacidad para captar la armonía del conjunto. "Die Verfasser solcher Geschichten lassen sich mit Tieren vergleichen, welche alle Töne einer Musik mit durchgehört haben, an deren Sinn aber das Eine, die Harmonie dieser Töne, nicht gekommen ist" ${ }^{20}$. En efecto, la historia de la filosofía se nos presenta desde ahora, muy en la línea que ya vimos en su filosofia de la historia, como una totalidad orgánica, «als ein organisch fortschreintendes Ganzes, als einen vernünftigen Zusammenhang" ${ }^{21}$.

El espíritu de la Ilustración aparece también aquí. Los filósofos, a quienes la opinión popular recluía en "su mundo" y de quienes los historiadores de ofi-

18 Einleitung in die Geschichte der Philosophie, editor Johanes Hoffmeister, Hamburgo, 1940. Hay edición castellana en Hegel, Introducción a la Historia de la Filosofia, traducción del alemán y prólogo de Eloy Terrón, Buenos Aires, 1956. A pesar de la calidad de esta traducción, se ha alterado aquí el orden de la edición alemana, suprimiéndose además el escrito de Heidelberg de 1816, el apéndice final y las notas críticas. Se han respetado en su integridad las características de la edición de Hoffmeister en su versión francesa: HEGEL, Leçons sur l'bistoire de la philosophie. Introduction: système et histoire de la philosophie, traducción de Jean Gibelin, París, 1970, 2 vols.

19 Einleitung in die Geschichte der Philosophie, edición J. Hoffmeister, pp. 5-6.

20 Ibid., p. 9.

21 Ibid., p. 11. 
cio prácticamente se olvidaban, son elevados por Hegel al rango de libertadores, de héroes de la razón pensante. "Was die Geschichte der Philosophie uns darstellt, ist die Reihe der edeln Geister, die Gallerie der Heroen der denkenden Vernunft, welche in Kraft dieser Vernunft in das Wesen der Dinge, der Natur und des Geistes, in das Wesen Gottes eingedrungen sind und uns den höchsten Schatz, den Schatz der Vernunfterkenntnis erarbeitet haben» ${ }^{22}$.

Mas es el historicismo hegeliano el que permite captar el sentido de las «hazañas del pensamiento».Y este es un punto central en su concepción de la historia de la filosofía. El trabajo colectivo de las generaciones anteriores toma cuerpo, consciente o inconscientemente, en nuestra actividad intelectual. Pues, en definitiva, nos caracterizamos por ser nosotros mismos un producto histórico: "...was wir sind, sind wir zugleich geschichtlich... Der Besitz an selbstbewusster Vernünftigkeit, welcher uns, der jetzigen Welt angehört, ist nicht unmittelbar entstanden und nur aus dem Boden der Gegenwart gewachsen, sondern es ist dies wesentlich in ihm, eine Erbschaft und näher das Resultat der Arbeit, und zwar der Arbeit aller vorhergegangenen Generationen des Menschengeschlechts zu sein» 23 .

"Diese Tradition ist aber nicht nur eine Haushälterin,die nur Empfangenes treu verwahrt und es so den Nachkommen unverändert überliefert. Sie ist nicht ein unbewegtes Steinbild, sondern lebendig, und schwillt als ein mächtiger Strom, der sich vergrössert, je weiter er von seinem Ursprunge aus vorgedrungen ist.

Der Inhalt dieser Tradition ist das, was die geistige Welt hervorgebracht hat, und der allgemeine Geist bleibt nicht stille stehen. Mit dem allgemeinen Geiste aber ist es wesentlich, mit dem wir es hier zu tun haben. (...) Der Geist der Welt aber versinkt nicht in diese gleichgültige Ruhe. Es beruht dies auf seinem einfachen Begriff. Sein Leben ist Tat” ${ }^{24}$. El pasado filosófico se contempla, pues, dialécticamente. no es un bloque de petrificada erudición sino un saber permanentemente renovado, un rio de vida. Esta imagen heraclítea, así como la contraposición entre erudición y saber, característica también del filósofo de Éfeso, reaparecen con fuerza en la dialéctica hegeliana. Si no todo el

22 Ibid., pp. 11-12, cursiva mía. Como se ve, la polémica antikantiana afecta también a la historia de la filosofía en general.

23 Ibid., p. 12, cursiva mía.

24 Ibid., p. 13. 
universo (ya aludí antes a su nada dialéctica visión de la naturaleza), ciertamente el ámbito del espíritu, en el que está incluida la filosofía, se distingue por su eterno movimiento, «su vida es acción".

Volvamos ahora a las lecciones sobre el concepto de filosofia según el texto preparado por el discípulo de Hegel y asiduo oyente de sus clases, Ludwig Michelet. Ellas constituyen el núcleo de esta "Introducción». Lo primero que se nos presenta es esta interesante observación metacientífica: carecen de relevancia los hechos históricos aislados de una totalidad. Como gustaba de repetir Ortega siguiendo a Hegel, la historia necesita de la teoría. "Además, ... hay que empezar por tener una visión general de conjunto antes de poder entrar en el detalle; de otro modo, los detalles nos impedirán ver el todo, los árboles nos impedirán ver la filosofía. (...) En ningún campo se confirma más esto que decimos que en el de la filosofía y en su historia. (...) Los hechos históricos sólo tienen un relieve, una significación, cuando se los pone en relación con un algo general y a través de su entronque con ello; tener ante los ojos este algo general es, por tanto, comprender la significación de los hechos en la historia” ${ }^{25}$.

\section{Concepto hegeliano de Historia de la filosofía}

E inmediatamente Hegel entra a considerar el concepto de historia de la filosofía. La unión en ésta de dos elementos antagónicos, lo histórico y lo filosófico, le lleva a descubrir una contradicción interna: "la filosofía se propone conocer lo que es inmutable, eterno, lo que existe en y para sí; su mira es la verdad. La historia, en cambio, narra lo que ha existido en una época para desaparecer en otra, desplazado por algo distinto" ${ }^{26}$. Ya veremos más adelante cómo su concepción dialéctica de la filosofía y su filosofia de la historia le permitirán resolver tal contradicción. Tampoco resulta alentadora la comparación de la filosofía con otras esferas del conocimiento humano. Así, a diferencia de la religión, «a la filosofía no se le reconoce por contenido ...una verdad fija y determinada de antemano". $Y$ a diferencia de otras ciencias, no progresa por vía de yuxtaposición. «La historia de la filosofía, en cambio, no revela ni la persistencia de un contenido simple, exento de adiciones, ni el simple proceso de

25 Lecciones sobre la bistoria de la filosofia, traducción de Wenceslao Roces, cit., vol. I, p. 12.

26 Ibid., p. 14. 
una pacífica acumulación de nuevos tesoros a los ya adquiridos con anterioridad, sino que parece ofrecer, más bien, el espectáculo de cambios incesantemente renovados del todo, sin que entre ellos subsista, a la postre, ni el nexo de unión de una meta común” ${ }^{27}$.

Hegel asume a veces en su análisis (y en esto recuerda a Aristóteles) el punto de vista vulgar para, a través de su refutación, intentar un avance en el plano de la teoría. Recoge, de este modo, la opinión corriente según la cual la historia de la filosofía consiste en una «galería de las necedades o, por lo menos, de los extravíos del hombre que se adentra en el pensamiento y en los conceptos puros» ${ }^{28}$. E incluso reconoce que muchas historias de la filosofía aceptan semejante prejuicio. Pero si lo admitimos, ese pretendido tejer y destejer de la filosofía nos llevará necesariamente a negar validez a tan absurdo trabajo. "Según esto, la historia de la filosofía no sería otra cosa que un campo de batalla cubierto de cadáveres, un reino no ya solamente de individuos muertos, físicamente caducos, sino también de sistemas refutados, espiritualmente liquidados, cada uno de los cuales mata y entierra el que le precede» 29.

Se trata, pues, de cerrar el paso al escepticismo. En esta tarea interviene decisivamente su propia dialéctica. Hegel reconoce la diversidad de filosofías, pero rechaza que tal diversidad sea analizada mediante "dem abstrakten Gegensatze von Wahrheit und Irrtum». En otro lugar se había burlado con mucha gracia de la vana pretensión de aquella señora que, por prescripción médica, acude al mercado pidiendo fruta y ante la respuesta de si lo que quería eran cerezas, ciruelas o uvas, contesta contrariada que no, que lo único que desea comprar es simplemente fruta. Aquí se opone radicalmente a quienes "por asco a lo particular en lo que lo general cobra realidad", se vuelven ciegos para captar la verdad en la historia de la filosofía: «... por muy distintas que sean las filosofías, todas ellas tienen algo en común: el ser filosofía. Por tanto, quien estudie o profese una filosofía, siempre y cuando lo sea verdaderamente, profesará la Filosofía. (...) Es menester que comprendamos que esta variedad entre las muchas filosofías no sólo no perjudica a la filosofía misma - a la posibilidad de la filosofía-, sino que, por el contrario, es y ha sido siempre

27 Ibid., p. 16.

28 Ibid., p. 17.

29 Ibid., p. 22. 
algo sencillamente necesario para la existencia de la propia ciencia filosófica, algo esencial a ellam ${ }^{30}$.

Progresando en su análisis, Hegel se dispone a iluminar su concepción de la historia de la filosofía, y para ello se detiene en estos dos conceptos previos: el de desarrollo, Entwicklung, y el de lo concreto, das Konkrete. (Prefiero traducir Entwicklung como "desarrollo" o "despliegue", en lugar de "evolución" como hace W. Roces, para evitar así toda connotación biologicista, ajena al pensamiento de Hegel). La explicación del primero de ellos le permite caracterizar el despliegue del espíritu. «Aquello para lo que lo otro es, es lo mismo que lo otro; sólo así puede ocurrir que el espíritu viva consigo mismo al vivir en el otro. El desarrollo del espíritu consiste, por tanto, en que, en él, el salir fuera y el desdoblarse sean, al mismo tiempo, un volver a sín. Respecto a lo concreto, subraya que si bien la forma del pensamiento es algo abstracto, sin embargo «de suyo, la idea es algo esencialmente concreto, puesto que es la unidad de distintas determinaciones. (...) Cuando la verdad es abstracta, no es tal verdad” ${ }^{31}$. De la combinación de ambos conceptos se obtiene el movimiento de lo concreto, que es lo que Hegel iba buscando para alcanzar su siguiente y decisiva conclusión: "Pues bien, la filosofía es, por sí misma, el conocimiento de este desarrollo y, en cuanto pensamiento comprensivo, este mismo desarrollo pensante; cuanto más lejos llegue este desarrollo, más perfecta será la filosofía» ${ }^{32}$.

De tan importante logro conceptual, Hegel se eleva a formular estas tres tesis que contienen, en lo esencial, su concepto de historia de la filosofía:

1. «La filosofía es sistema en desarrollo y lo es también, por tanto, la historia de la filosofiam.

2. "La sucesión de los sistemas de la filosofía en la historia es la misma que la sucesión de las diversas fases en la derivación lógica de las determinaciones conceptuales de la idea. (...) Y a la inversa ... Podría pensarse que la filosofía tiene que seguir en las fases de la idea otro orden que aquel en que estos conceptos se manifiestan en el tiempo; pero, visto en su conjunto, el orden es el mismo... : de lo dicho se desprende que el estudio de la historia de la filosofía es el estudio de la filosofía misma y no podía ser de otro modo".

\footnotetext{
30 Ibid., pp. 23-24.

31 Ibid., pp. 28-29.

32 Ibid., p. 32, cursiva mía.
} 
3. "Sólo merece el nombre de ciencia (Wissenschaft) una historia de la filosofía concebida como sistema de desarrollo de la ideas ${ }^{33}$.

Es evidente que la validez de estas tesis historiográficas descansa, en última instancia, sobre el sistema hegeliano en su conjunto. Mientras aceptemos la filosofía de la historia y la dialéctica de Hegel, su concepción historiográfica se mantiene en pie. En caso contrario, caerá al suelo como castillo de arena. Algo de esto ha ocurrido en nuestra época cuando al desmantelamiento del hegelianismo sucedió un aprovechamiento fragmentario del tal legado, gracias al cual han podido salvarse algunas perlas especulativas y metodológicas. Ya veremos más adelante las huellas de esta herencia en el debate historiográfico contemporáneo.

Dilucidado el núcleo de su concepción de la historia de la filosofía, Hegel se dedica ahora a aplicarlo ${ }^{34}$. Estas son las principales consecuencias que extrae:

- El desarrollo de la filosofía en la historia viene marcado por la necesidad.

- Esa necesidad implica que ninguna filosofía es refutada por completo sino que se conserva como un momento de la totalidad. La consciencia de esa unilateralidad en la que se manifiesta su necesidad histórica - todo lo que nace debe perecer-, ha de impulsar al historiador de la filosofia a saber justificar las diversas filosofías. «Die Geschichte der Philosophie ist so die Rechtfertigung aller Philosophieen" ${ }^{35}$. De aquí extrae Hegel una útil propuesta metodológica: «la actitud que se adopte ante una filosofía deberá tener un lado afirmativo y otro negativo; sólo teniendo en cuenta ambos aspectos conjuntamente haremos justicia a la filosofía de que se trate. El lado afirmativo tarda siempre más en revelarse, lo mismo en la vida que en la ciencia; por eso el refutar es siempre mas fácil que el justificar”.

- Hay que considerar especialmente los principios, de cuyo desarrollo posterior surgen los sistemas filosóficos.

33 Ibid., pp. 32-35. Las limitaciones del historicismo aquí expuesto y la preeminencia de lo lógico en la dialéctica hegeliana han sido subrayadas en un interesante articulo de JindOich Zelený titulado "A propósito de la relación dialéctica entre lo lógico y lo histórico", en Dialéctica y conocimiento, traducción de Jacobo Muñoz, Madrid, 1982, pp. 52-81.

34 Ibid., pp, 40-42.

35 Einleitung in die Geschichte der Philosophie, Anhang 2, p. 280, cursiva mía. 
- La historia de la filosofía estudia el pensamiento vivo. Su tarea no consiste en la visión del pasado filosófico como objeto de erudición arqueológica. "El contenido de esta historia son los productos científicos de la razón, que no son perecederos ni se incorporan al pasado. En este campo se cultiva lo verdadero, y lo verdadero es eterno, no existe en una época para dejar de existir en otra... La filosofía no tiene nada de sonambulismo, sino que es, por el contrario, la más despierta de las conciencias. (...) Estos conocimientos son, por ello mismo, algo más que pura erudición, algo más que el conocimiento de lo muerto, lo enterrado, lo descompuesto; la historia de la filosofía ha de ocuparse de lo que no envejece, de lo presente vivon.

Finalmente, Hegel traza un estrecho paralelo entre la historia de la filosofia y la filosofía misma. Sus dos principales conclusiones son:

1. «Las primeras filosofías son las más pobres y las más abstractas de todas». (Algo que ya había observado Aristóteles al referirse a los presocráticos).

2. «Es necesario abordar el problema históricamente; es decir, atribuir a las filosofías solamente lo que sabemos que encerraban y no lo que a nosotros nos parezca obligado que contuvieran» ${ }^{36}$. O sea, una cautela metodológica que siempre deberíamos tener presente. Hegel concluye este apartado de la "Introducción», y con él prácticamente su reflexión sobre el concepto de la historia de la filosofía, en su mejor línea historicista. «Toda filosofía es la filosofía de su tiempo, un eslabón en la gran cadena del desarrollo espiritual; de donde se desprende que sólo puede dar satisfacción a los intereses propios de su tiempo" ${ }^{37}$.

$\mathrm{Al}$ término de sus lecciones, es decir, una vez verificada en la historia su original concepción de la historia de la filosofía, labor que todavía hoy nos llena de admiración como hace más de 150 años a los universitarios alemanes de la época, Hegel ofreció esta síntesis de su aportación historiográfica que vale la pena reproducir ahora. «El resultado general de la historia de la filosofía es éste: en primer lugar, que no ha existido en todo tiempo más que una filosofía, cuyas diferencias coexistentes representan otros tantos aspectos necesarios de un solo principio; en segundo lugar, que la secuencia de los sistemas filosófi-

36 Ibid., pp. 43 y 45.

37 Ibid, p. 48. 
cos no es una sucesión fortuita, sino la sucesión necesaria del desarrollo de esta ciencia; en tercer lugar, que la filosofía final de una época es el resultado de este desarrollo y la verdad en la forma más alta que acerca de sí mismo alcanza la conciencia de sí del espíritu» ${ }^{38}$.

\section{Críticas a la concepción hegeliana de la Historia de la filosofia}

Gracias a Hegel la historia de la filosofía alcanzó su pleno reconocimiento como disciplina académica y se convirtió en centro de interés tanto de historiadores como de filósofos. Pero una historiografía tan vinculada al sistema hegeliano no podía permanecer indemne a los avatares de éste. A la ambigüedad de ese ingente legado, puesta de relieve en la oposición de tendencias surgidas en su seno, siguió la aparición de escuelas de pensamiento nacidas en polémica frontal con él. La herencia especulativa de Hegel, incluida su filosofía de la historia, quedó así dispersa. En la segunda mitad del siglo XIX vemos ya fragmentado y ruinoso el hasta entonces aparentemente sólido edificio. Ello no significaba, por supuesto, que su contribución historiográfica cayera en el olvido. De hecho, ocurrió más bien al contrario. Bien reclamándose herederos de él, bien definiéndose como sus irreductibles adversarios, ya en forma expllcita o implícita, toda concepción de la historia de la filosofía elaborada tras la muerte de Hegel refleja el eco de su teoría.

De las insuficiencias de la concepción hegeliana sacadas a la luz por la historiografía posterior, resulta imposible hacer un balance global en estas páginas. Baste, pues, recoger aquí algunas críticas especialmente relevantes. En primer lugar, Dilthey. "La comprensión filosófica a base de la idea de desarrollo ha sido lograda por vez primera por G.W.F. Hegel en sus Vorlesungen über die Geschichte der Philosophie. Sin embargo, Hegel desdeñó la base rigurosa de los métodos filológicos y estableció entre los sistemas una conexión evolutiva deficiente porque se valió de relaciones lógicas completamente abstractas». El mundo histórico es más justamente valorado en la propuesta diltheyana: "en oposición con la actitud de Hegel, no se explica el desenvolvimiento de la filo-

38 Ibid, vol. III, pp. 517-518. Sobre la contribución de Hegel a la historiografía filosófica puede verse la obra colectiva Hegel and the History of Philosophy, La Haya, 1974, que incluye una amplia bibliografía. 
sofía en virtud de las relaciones que mantienen entre sí los conceptos en el pensamiento abstracto, sino en virtud de los cambios que se realizan en el hombre entero según su vida y realidad plenas. Por esto se procura averiguar la conexión causal que los sistemas filosóficos han mantenido con la totalidad de la cultura y con la cual ha influido sobre ésta» ${ }^{39}$.

Este eclipsamiento de la historia debido a la hegemonía de la lógica fue subrayado asimismo con fuerza por otro gran filósofo europeo contemporáneo, continuador en Italia del idealismo alemán y también excelente historiador de la cultura, Benedetto Croce. En su burla de la línea puramente especulativa seguida por ciertos hegelianos, se muestra la insatisfacción de una crítica histórica que no estaba ya dispuesta a silenciar por más tiempo la estrechez de un método errado. "Quella stessa filosofia, che, in forza di una sua dottrina logica, aveva con tanta efficacia rivendicato il valore della storia, delle res gestae, per effetto di un'altra sua dottrina logica era poi messa nell'impossibilità di riconoscere il valore della historia rerum gestarum, e quindi delle stesse res gestae. Affamata di storia, nutrita di storia, la filosofia di Hegel faceva poi, senza rendersene ben conto, la propaganda del digiuno. E la contradizione si manifestò alla luce del sole, agli occhi di tutti; giacché, come dalla scuola di Hegel uscí una serie di grandi scrittori di storie, dalla stessa scuola vennero fuori i piú petulanti e comici dispregiatori della storia e dei fatti, che si siano mai visti al mondon ${ }^{40}$.

Pero la crítica más radical a la filosofía de la historia hegeliana, y también la más antigua, pues se comenzó a redactar en 1845 , procede de los fundadores del marxismo. En su esfuerzo por fundamentar la concepción materialista de la historia que estaban elaborando, Marx y Engels polemizaron acremente con la filosofía alemana de la época. Una vez conseguido su principal propósito de aclararse a sí mismos las ideas, confiaron el manuscrito - según la conocida expresión de Marx - «a la roedora crítica de las ratas»: La Ideología Alemana no apareceria publicada hasta... 1932. Se le reprocha a Hegel, ante todo, el haber reducido el mundo histórico a una mera construcción especulativa, ignorando así la base real de la historia. "No se trata de buscar una cate-

39 W. DiltheY, Historia de la Filosofla, traducción de Eugenio Imaz, México D.F., 1979, 2.2 ed., pp. 11-12.

40 BENEDETTO CROCE, "Cid che è vivo e ciò che è morto della filosofia di Hegel", en Saggio sullo Hegel e altri scritti di storia della filosofia, Bari, 1967, 5.2 ed., p. 98. La primera edición de este famoso ensayo crociano es de 1906. 
goría en cada período, como hace la concepción idealista de la historia, sino de mantenerse siempre sobre el terreno histórico real, de no explicar la práctica partiendo de la idea, de explicar las formaciones ideológicas sobre la base de la práctica material. (...) Y, mientras que los franceses y los ingleses se aferran, por lo menos, a la ilusión política, que es ciertamente la más cercana a la realidad, los alemanes se mueven en la esfera del 'espíritu puro' y hacen de la ilusión religiosa la fuerza motriz de la historia. La filosofía hegeliana de la historia es la última consecuencia, llevada a su 'expresión más pura', de toda esta historiografía alemana, que no gira en torno a los intereses reales, ni siquiera a los intereses políticos, sino en torno a pensamientos puros" ${ }^{41}$. La pretensión hegeliana de crear una historia de la filosofía autónoma se convertiría, por tanto, en vana ilusión, ya que, en definitiva, es la vida la que determina la conciencia y no al revés. "La moral, la religión, la metafísica y cualquier otra ideología y las formas de conciencia que a ellas corresponden pierden, así, la apariencia de su propia sustantividad. No tienen su propia historia ni su propio desarrollo, sino que los hombres que desarrollan su producción material y su intercambio material cambian también, al cambiar esta realidad, su pensamiento y los productos de su pensamiento. No es la conciencia la que determina la vida, sino la vida la que determina la conciencia»" ${ }^{42}$.

Desde otra perspectiva, un estudioso francés ha observado la huella de Winckelmann en la historiografía hegeliana. Tal concepción del arte que entusiasmaba a Herder, fue asimilada y desarrollada posteriormente por Hegel. En él, la historia nos hará conocer temporalmente y a posteriori lo que el espíritu, en que reside la esencia de toda filosofia, asume intemporalmente y a priori. De aquí, la noción hegeliana de un desarrollo intemporal del espíritu que es el alma del desarrollo temporal de la historia ${ }^{43}$.

Por su parte, un maestro italiano de la historiografía filosófica, Rodolfo Mondolfo, ha explicado cómo para lograr un desarrollo teórico se necesitan condiciones preparatorias que, siendo necesarias, no siempre son suficientes. En esta posibilidad de insuficiencia consiste precisamente la contingencia. Y ésta se relaciona con los factores individuales que juegan su papel con respec-

41 Carlos MarX-Federico Engels, La Ideologla Alemana, traducción de Wenceslao Roces, Barcelona, 1970 , pp. 40 y 42.

42 Ibid, p. 26.

43 MARTLAL Gueroult, "Le problème de la légitimité de l'histoire de la philosophie», en AA.VV., La philosophie de l'histoire de la philosophie, París, 1956, pp. 45-68. 
to a las condiciones históricas del ámbito intelectual. Habría que evitar dos errores opuestos: el de considerar la historia de la filosofía como una sucesión incoherente de sistemas que se contradicen, y el de contemplar este desarrollo histórico dominado por una necesidad interior, absoluta, como defendía Hegel ${ }^{44}$.

Para terminar esta referencia a la recepción crítica del concepto de historia de la filosofía elaborado por Hegel, podemos recordar el juicio general bastante ecuánime de un estudioso español. «El influjo hegeliano en lo historiográfico tiene muchos matices, pues no se lo debe admitir únicamente en los secuaces declarados, ceñidos a su esquema dialéctico, sino también en cuantos, siguiendo su ejemplo, refuerzan en demasía el momento especulativo en la comprensión de las series y los nexos y encauzan los contenidos históricos según un 'constructivismo' más o menos preconcebido y deformador" "5.

Después de Hegel, y vistos los resultados, nadie intentó levantar de nuevo un edificio teórico que, a semejanza del pensador alemán, contuviera al mismo tiempo un sistema filosófico original, una filosofía de la historia, una concepción de la filosofía y de su historia, e incluso una extensa historia de la filosofía. Los objetivos fueron más modestos desde entonces; y, salvo en el caso de ciertos post-hegelianos, se prestó una mayor atención al método histórico en detrimento del constructivismo especulativo anterior. Un fruto de la madurez de la historiografía filosófica del siglo XIX lo encontramos en la magnífica reconstrucción del pensamiento griego de Eduard Zeller: Die Philosophie der Griechen in ibrer geschichtlichen Entwicklung dargestellt, Tubinga, 1855-1868. Aunque el impulso historiográfico procede de Hegel, vemos ya aquí una metodología propia liberada de todo apriorismo.

Otra vía de avance, ya alejada del espíritu especulativo hegeliano, procedió del campo filológico. A lo largo del siglo XIX y hasta los primeros años del siglo $\mathrm{XX}$, fueron editándose excelentes ediciones críticas de los filósofos griegos, todavía hoy indispensables. Un lugar de honor en esta gigantesca tarea correspondió a los helenistas alemanes. Señalemos algunos hitos en este camino:

44 Rodolfo MONDOLfo, Problemas y métodos de investigación en la historia de la filosofia, Buenos Aires, $1969,4 .^{2}$ ed., cap. IV.

45 FRANCISCO ROMERO, "La historia de la filosofía según Hegel», en La estructura de la historia de la filosofia y otros ensayos, Buenos Aires, 1967, p. 167; el estudio sobre la historiografía hegeliana ocupa las pp. 159-172. 
Aristotelis Opera, edición de la "Academia Regia Borussica", cuyos dos primeros volúmenes con el texto de las obras aristotélicas fue preparado por Immanuel Bekker y se publicó en Berlín el año 1831; Hermann Diels, Doxographi Graeci, Berlín, 1879, obra fundamental para el estudio de las fuentes de la filosofía griega; Hermann Usener, Epicurea, Leipzig, 1887, de gran interés por la amplísima recopilación de fragmentos epicúreos; Platonis Opera, edición de John Burnet, Oxford, 1892-1906, en cinco volúmenes, que aun sigue en pie; y la ya histórica antología de los presocráticos de Hermann Diels, Die Fragmente der Vorsokratiker, Berlín, 1903. Ello permitió que el lenguaje de los filósofos griegos pasara a tener una importancia de primer orden en las investigaciones historiográficas, algo de especial relevancia, pues, como ha escrito Kurt von Fritz, «de entre todos los pueblos europeos, es el griego el único que ha sacado de sí mismo el lenguaje filosófico y científicon. La acentuación y profundización de esta línea renovadora ha traído consigo un peso creciente de la hermenéutica dentro de la filosofía contemporánea desde mediados del siglo XX. 